\title{
The difference of tensile bond strength between total and self etch adhesive systems in dentin
}

\author{
Selly Yusalina, Moch. Richata Fadil, Milly Armilya Andang \\ Department of Conservative Dentistry Faculty of Dentistry Universitas Padjadjaran
}

\section{ABSTRACT}

Total etch adhesive system has been widely used in teeth conservation area as an adhesive agent before implicating composite resin restoration agent. The aim of this research is to prove the difference of tensile bond strength between total etch (Single Bond) and self etch adhesive system (Adper prompt L-Pop) on dentin surface in vitro. The extracted and non carries maxillary premolar teeth were used in this research and were divided into 2 groups. The first group comprised 15 specimen teeth etched in phosphoric acid and was applicated with the Single Bond adhesive agent. The second group comprised 15 specimen teeth, applicated with the Adper Prompt-L-Pop. The composite resin (Z 350, 3M) was applied incrementally and each of the layers was rayed for 20 seconds. The specimens were stored in physiologic solution before they were tested. Tensile bond strength was measured by LRX Plus Lloyd Instrument, with $1 \mathrm{~N}$ load and $1 \mathrm{~mm} /$ minute speed, and the measurement result was in Mpa unit. The result was evaluated statistically by the Student $\mathrm{t}$-test with $\mathrm{a}=0.05$. Single Bond (the $5^{\text {th }}$ generation) showed a better bond strength compared to the Adper Prompt-L-Pop (the $6^{\text {th }}$ generation).

Key words: Adhesive systems, tensile bond strength.

\section{INTRODUCTION}

The use of composite resin is getting more popular because it has a better ability compared to the conventional amalgam restoration. Every teeth restoration demands retention through some adhesive systems. Dental adhesives were improved to give a strong interfacial bond between teeth substrate and restoration agent used in teeth conservation area. Sometimes interfacial bond failure between teeth substrate and restoration agent happens when both have difference mechanical characters. Adhesive between composite resin and dentin is harder to get, especially because of the structure and the composition of dentin. Enamel almost all comprises of inorganic agent while dentin is a live tissue comprises inorganic compounds (45\%), organic compounds (33\%) and water. ${ }^{1}$

Dentin tubules are filled by processus odontoblast and dentin fluid which are the main parts of dentin. This is a significant difference compared to enamel since dentin is characterized by the intrinsic fluid so the adhesive agent should be hydrophilic in order to get in and be able to have a contact with the dentin. ${ }^{2}$ When dentin is instrumented mechanically, a debris layer on the surface, called smear layer, will fill dentin orifice tubules so it reduces dentin permeability. ${ }^{3}$ Bonding mechanism in dentin will be effective if the smear layer is totally dissolved, the intertubular and peritubular are dissolved, the collagen fiber is 
opened and after the resin monomer infiltrated, the hybrid layer is formed. ${ }^{4}$

The difference between dentin bonding system generation is based on the handling of smear layer. In the first and second generations, the aim is to get an adhesive chemistry between adhesive agents and smear layer, in order to protect teeth pulp. Smear layer attaches on teeth surface in superficial area so the adhesive agent obtained is not really good. In order to enhance the bond strength of dentin adhesive, primer solution can change smear layer into the third generation adhesive system. In the fourth generation system, smear layer is totally disposed with the organic acid etching, followed by primer and adhesive applications which form hybrid layer so high quality bond strength can be obtained.

Enamel and dentin etching are the basis of the fourth and fifth generations of enamel-dentin adhesives which are mostly used recently. That is why this adhesive is commonly called total etching system. Adhesive system with total etch is a single dosage contains primer and adhesive agent which needs phosphoric acid etching procedure before. The last improvement was self etching adhesive system which mixes acid conditioning with priming and bonding, so there is no need to do etching before. Self etching adhesive system produces equal enamel bond strength with the total adhesive system, but some previous in vitro researches showed the dentin bond strength was significantly lower than the one obtained by total etch adhesive system. ${ }^{3}$

Based on the statement before, a research should be conducted in order to find out the difference of tensile bond strength between dentin bonding with total etch adhesive system and self etching adhesive system.

\section{MATERIALS AND METHODS}

Thirty maxillary premolar teeth with carries free extracted the last one month were taken randomly from the population. They were cleaned by the ultrasonic cleaner and stored in distilled water. The roots of the teeth, more or less half part of them, were cut by a diamond disc in order to get specimens with the crown size to the root equals 1 to 1 . Buccal surface was grinded using the diamond disc with water cooler in a handpiece motor until the enamel area disappears. Level it by 600 grit-Sic paper to make even dentin surface. The preparation should be on the dentin superficial layer (more or less $1 \mathrm{~mm}$ from DEJ).

There were 30 samples in this research. They were then divided into two groups; each group consisted of 15 teeth each for total etching adhesives system group and self adhesives system group.

First group of teeth was total etch adhesive system group. Dentin surface was cleaned and dried using a wind spray. Thirty five percent of phosphoric acid was applied to the dentin specimens for 15 seconds and rinsed for 10 seconds. Dry with a mild blast for 5 seconds to keep the dentin moist. Single Bond adhesive applicated on the etched dentin surface using an applicator, then it was sprayed by mild air for 2-5 seconds, and rayed by LED for 10 seconds.

Second group was group of teeth with selfetch adhesive system. Material on the red reservoir was pressed so that the liquid would move into the yellow reservoir (middle). Next, the yellow reservoir was pressed so that the liquid would flow to the green reservoir. Let it stand for 5 seconds so that the adhesive material applicator could wet the applicator perfectly. The dentin surface was cleaned and dried using a spray wind until the dentin looked shiny or moist. Then the applicator was applied to the dentin for 15 seconds. Spray lightly to the adhesive material so a thin layer was formed, then it was rayed for 10 seconds.

Specimens from each group were stored separately and the following procedure was performed to both groups. Matrix was made of metal with size of $4 \times 4 \times 5 \mathrm{~mm}$, and placed on the dentin. Composite resin with $2 \mathrm{~mm}$ thickness was applied, condensed and rayed for 20 seconds. The composite used in this study was Z350 3M ESPE. The resin composite was added incrementally up to $5 \mathrm{~mm}$ high and rayed back for 20 seconds. The specimens were kept in self-curing acrylic resin (buried at height $\pm 2 \mathrm{~mm}$ ). All specimens were immersed in $0.9 \%$ physiological saline for 24 hours.

The specimens' tensile strength of group I and II were then measured using tensile strength test machine (LRX Plus Llyoid Instruments) at speed up to $1 \mathrm{~mm} /$ minute until a fracture occurred and the data during the fracture was recorded for statistical test. 


\section{RESULTS}

From 15 samples in the two test groups resulted the mean of tensile bond strengths using total etch adhesive system was $1.5689 \mathrm{MPa}$ and the mean of tensile bond strength with self-etch adhesive system was $0.7291 \mathrm{MPa}$. These results indicated the mean difference of the tensile bond strength. The mean of tensile bond strength of total etch adhesive system group was greater than the mean of the tensile bond strength of self etch adhesive system.

The research results were then analyzed using the student-t test with the $95 \%$ level of significance. From that statistical calculation test results showed a significant difference. The difference between the tensile bond strength value of the mean nano composite using a total etch adhesive system was greater than the mean tensile bond strength of self adhesive adhesive system on dentin surface.

\section{DISCUSSION}

In this study, test on the tensile bond strength of composite resin to dentine was performed in order to see the difference in bond strength between two types of adhesive systems. In this study, tooth preparation was conducted approximately $1 \mathrm{~mm}$ below DEJ and was done to the teeth aged $15-25$ years, due to the more superficial dentin has fewer tubule with a smaller diameter. Deeper dentin has more tubule with larger diameter and more fluid. ${ }^{5}$

Pashley $^{6}$ reported the density of tubule and peritubular dentin area increased towards the pulp and peritubular dentin area decreased towards the pulp, so that the water content and permeability of the dentin were not the same for all regions because the variations of the number of tubule per mm. ${ }^{2}$ As age grows, there is much mineralization in dentin, the ratio of peritubular/ intertubular dentin tubule increased and the amount of dentin tubule decreased. ${ }^{5}$

In this study, tensile bond strength tests were performed on dentin specimen surface which was cleaned in the same manner. Bond strength measurements were conducted after it was stored in physiological saline, although the critical period to evaluate this restorative system was immediately after the placement of composites.

The first group used the Single Bond (Total Etch Adhesives system) which was the fifth generation bonding agent that has a two-step application. The teeth were etched and followed by the application of bonding agent, which contained a mixture of primer and adhesive in one bottle. The moist nature of dentin surface was a critical factor for the success of dentin bonding. If the surface was too dry, the collagen and dentin demineralized on vital teeth would collapse, whereas if it was too wet, there would be phase changes in resin made of ethanol or acetone and water bubbles which was able to reduce the bond strength would be formed. ${ }^{7}$ The second group using Prompt Adper L-Pop (Self-Etching adhesive system) which combined acid conditioning with priming and bonding, so etching did not need to be conducted in the first place.

The effective bond strength of dentin could be achieved if the adhesive system produced a layer which was the mixture of resin monomers and organic dentin (collagen fibers), which was known as the zone hybridization. The quality of varies depending on the hybrid layer depended on the etch and $\mathrm{pH}$, the ability of resin monomer to penetrate into the demineralized dentin, morphologic and chemophysiologic dentin characteristics. In the image of SEM Adper Prompt L-Pop, the hybrid layer formed was very thin, thus it affected the bond strength between composite and dentin. ${ }^{8}$

Application of thirty five percent of phosphoric acid application to the first group produced superficial dentin demineralization, removing smear layer and opening the collagen fibers from the dentin matrix in vital tooth, in order to support the resin monomer infiltration. ${ }^{7}$ Hybrid layer formation with the absorption of resin material into the collagen tissue was opened after the acid etching on the dentin was considered to create an adequate bond between resin and dentin. Imperfect resin absorption collagen from the collagen tissue was also caused by the demineralized dentin zone that resulted bond strength reduction. This might be seen from the value of tensile bond strength (TBS) which was higher in Group 1 (mean of TBS Single Bond: $1.5689 \mathrm{MPa}$ ) compared to Group 2 (TBS mean Adper Prompt L-Pop: $0.7291 \mathrm{MPa})^{7}$ 
Some studies claimed that there was a correlation between hybrid layer thicknesses with bond strength, while others claimed that the hybrid layer thickness did not play a role in bond strength. Further research on the relationship of the bond strength with hybrid layer thickness needs to be conducted in order to reach concrete conclusions. ${ }^{9}$

Tensile bond strength of total etch adhesive system on dentin was higher than self-etching adhesive systems associated with the facts that the total etching removed the smear layer completely, opened the collagen fibers as mentioned above, so that the resin monomers total etch adhesive system could soak into the enamel substrate or dentin which was cleaned and demineralized, while self-etching adhesive systems did not reach the same demineralization depth due to the integral smear layer. ${ }^{10}$

As smear layer played an important role in bonding. Normal smear layer thickness was 0.5$5.0 \mu \mathrm{m}$, which acted as a barrier that reduced the permeability of dentin. This was considered as a barrier that must be eliminated so that the resin can be bonded to the dentin substrate below. ${ }^{11}$

The result study of Vasconcellos et al. ${ }^{10}$ also showed that the TBS total-etching adhesive systems was higher than the self-etching adhesive systems and had higher TBS in the enamel than in the dentin. This can be attributed to the fact that the total-etching completely removed smear layer and opened the collagen fibers as mentioned before. ${ }^{11}$

This research also correlated with previous studies conducted by Spohr and Sobrinho ${ }^{12}$ Single bond (total-etching adhesive systems) has the highest score of tensile bond strength than the $6^{\text {th }}$ generation of self-etching adhesives both for tensile and shear test tests. ${ }^{12}$ Good adhesion was ideally shown by high tensile bond strength and therefore it possessed a high resistance against microleakage at the corner of enamel cavity and dentin. Another factor that caused low bond strength in both group was because both of the bonding agent contained HEMA and water. Dentin humidity varied between $1 \%$ in superficial dentin, and approximately $22 \%$ in the inner dentin..$^{13}$ If the etched cavity was dried in order to evaluate the chalky image in enamel, and then water was added for rehydration and developed the collagen fibers in the dentin, superficial rehydration might be well occurred, but then it became water excess in some areas in the inner dentin. ${ }^{13}$ There was much water in the tubule filled with dentin fluid, so the accumulated globuli on the tubule wall was formed, reduced the tubule's permeability, allowing the primary application, respectively for tubule dehydration to form the normal resin tag.

Optimum dentin humidity was important for the success of composite attachment to dentin. If it was too moist, water bubbles could not be formed on dentin and adhesive borders, which caused imperfect interface hybridization. For hydrophilic resin primer, to make the demineralized dentin penetration went perfectly; the solvent taking primer should also be evaporated. Alcohol and acetone solvents evaporated faster than water solvent. Due to higher water content, this material was more sensitive to water content of the demineralized dentin, especially during the polymerization and hybrid layer formation. ${ }^{8,13}$

Inappropriate water elimination from the collagen tissue was the result of a combination of monomers and the remaining water in the demineralized dentin may hamper the polymerization of bonding agent. In the clinical situation, we must remove the remaining water until the teeth condition looked moist, and did not show the white color. Water excess could also dilute the primer and caused the bonding agent less effective. Water remain inhibited the polymerization of bonding agent and could cause a drop in bond strength as shown in this study. The use of incorrect wet bonding technique was the other factor that affected the bond strength of Single bond. ${ }^{8}$

\section{CONCLUSION}

Based on the research and the statistical analysis conducted, a conclusion can be drawn as follows: the tensile bond strength of a total etch adhesive system is higher than the self-etching adhesive systems when used in nano composite restoration. The ties of dentin bonding with total etchs adhesive system is stronger with dental tissue compared to self-etching adhesive system. 


\section{REFERENCES}

1. Demarco FF, Turbino ML, Matson E. Tensile bond strength of two dentin adhesive systems. J Braz Dent 1998;9(1):11-8.

2. Roulet JF, Degrange M. Adhesion the silent revolution in dentistry. Illionis: Quintessence Publishing Co; 2000. p. 34-5.

3. Roberson TM, Heymann HO, Swift EJ. Studervant's art and science of operative dentistry. St. Louis: Mosby Inc.; 2006. p. 24050,476-82.

4. Kugel $G$, Ferrari M. The science of bonding: from first to sixth generation. JADA 2000; 131:21-4.

5. Yesilyurt C, Bulucu B. Bond strength of totaletch dentin adhesive systems on peripheral and central dentinal tissue: A microtensile bond strength. J Contemporary Dent Practice 2006;7(2):2-11.

6. Pashley $\mathrm{DH}$. The evolution of dentin bonding from no-etch to total-etch to self-etch. [cited 2009 Oct 19]. Available from:http//www. kuraraydental.com/newsletter/ats-premier. pdf.

7. Neelima L, Sathish ES, Bupesh DK. Evaluation of microtensile bond strength of total-etch, self-etch, and glass ionomer adhesive to human dentin: An in vitro study. Indian J Dent
Res 2008;19:129-33.

8. Mousavinasab SM, Farhadi A, Shabanian M. Effect of storage time, thermocycling and resin coating on durability of dentin bonding systems. J Dent Res 2009;6(1):29-37.

9. Dhawan R, Indira R, Dhawan S. A comparative evaluation of tensile bond strength and scanning electron microscopic study of three generation bonding agents. An in-vitro study. J Conserv dent 2005; (8)1:8-18.

10. Vasconcellos WA, Susin AH, Vaz LG, Oliveira Jr OB. Tensile bond strength: Evaluation of four current adhesive systems in abraded enamel and deep dentin. J Indian Prosthod Soc 2007;7(June):77-8.

11. Ozok AR,Wu MK, De Gee AJ, Wesselink PR. Effect of dentin perfusion on the sealin ability and microtensile bond strength of a totaletch versus an all in-one adhesive. Dent Mater 2004;20:479-86.

12. Spohr, Sobrinho. In: Ozok AR,Wu MK, De Gee AJ, Wesselink PR. Effect of dentin perfusion on the sealin ability and microtensile bond strength of a total-etch versus an all in-one adhesive. Dent Mater 2004;20:479-86.

13. Nakabayashi N, Pashley DH. Hybridization of dental hard tissues. Tokyo: Quintessence Publishing Co., Ltd; 1998. p. 65-77. 